\title{
Can a telemonitoring system lead to decreased hospitalization in elderly patients?
}

Johan Lyth, Leili Lind, Hans L. Persson and Ann-Britt Wiréhn

The self-archived postprint version of this journal article is available at Linköping University Institutional Repository (DiVA):

http://urn.kb.se/resolve?urn=urn:nbn:se:liu:diva-159582

N.B.: When citing this work, cite the original publication.

Lyth, J., Lind, L., Persson, H. L., Wiréhn, A., (2019), Can a telemonitoring system lead to decreased hospitalization in elderly patients?, Journal of Telemedicine and Telecare, , UNSP

1357633X19858178. https://doi.org/10.1177/1357633X19858178

Original publication available at:

https://doi.org/10.1177/1357633X19858178

Copyright: SAGE Publications (UK and US)

http://www.uk.sagepub.com/home.nav 


\section{Can a telemonitoring system lead to decreased hospitalization in elderly patients?}

J. Lyth ${ }^{1}$, L. Lind ${ }^{2,3}$, H.L. Persson ${ }^{4}$, A.B. Wiréhn ${ }^{1}$

${ }^{1}$ Research and Development Unit in Region Östergötland and Department of Medical and Health Sciences, Linköping University, Linköping, Sweden

${ }^{2}$ Department of Biomedical Engineering/Health Informatics, Linkoping University, Linköping, Sweden

${ }^{3}$ RISE Research Institutes of Sweden AB / Division ICT SICS East, c/o Linköping University, Linköping, Sweden

${ }^{4}$ Department of Respiratory Medicine, Department of Medical and Health Sciences, Linköping University, Linköping, Sweden.

Correspondence to: Johan Lyth, Research and Development Unit in Region Östergötland, SE-581 85

Linköping, Sweden

Phone: +46-10 10387 83, Fax: +46-10 1038501

E-mail: johan.lyth@regionostergotland.se

The Authors declares that there is no conflict of interest 


\begin{abstract}
Introduction: Growing populations of elderly patients with chronic obstructive pulmonary disease (COPD) or heart failure (HF) require more healthcare. A 4-year telehealth intervention - the Health Diary system based on digital pen technology - was implemented. We hypothesized that study patients with advanced COPD or HF would have lower rates of hospitalization when using the Health Diary. The aim was to investigate the effects of the intervention on healthcare costs and the number of hospitalizations, as well as other care required in COPD and HF patients.
\end{abstract}

Methods: Patients were introduced to the telemonitoring system which was supervised by a specialized hospital-based home care (HBHC) unit. Staff associated with this unit were responsible for the healthcare provided. The study included patients with COPD or $\mathrm{HF}$, aged $\geq 65$ years who were frequently hospitalized due to exacerbations - at least two inpatient episodes the last twelve months. Observed number of hospitalizations and total healthcare costs were compared with the expected values, which were calculated using the generalized estimating equations (GEE) method.

Results: A total of $36 \mathrm{COPD}$ and $58 \mathrm{HF}$ patients with advanced stages of disease were included. The number of hospitalizations was significantly reduced for both HF and COPD patients participating in telemonitoring. Accordingly, hospitalization costs were significantly reduced for both groups, but the total healthcare cost was not significantly different from the expected costs.

Conclusion: A telemonitoring system, the Health Diary, combined with a specialized HBHC unit significantly decreases the need for hospital care in elderly patients with advanced HF or COPD without increasing total healthcare costs. 
Keywords: Home telecare, Telemedicine, Telecare, Telehealth, Digital pen, Heart Failure, Chronic obstructive pulmonary disease

\section{Introduction}

Populations of elderly patients with advanced heart failure (HF) and chronic obstructive pulmonary disease (COPD) are growing. In Sweden, the prevalence of HF is estimated at $10 \%$ for those $\geq 80$ years old $^{1}$, and the prevalence of COPD is already $8 \%$ at 50 years, and increases by age ${ }^{2}$. Dyspnea is a characteristic feature of both diseases, which initially is exertional and, as the diseases progress, appears at rest as well. Edema is associated with $\mathrm{HF}$, and patients with COPD demonstrate chronic cough which often is associated with increased mucus production. Both diseases may demonstrate episodes of worsening symptoms, known as exacerbations. ${ }^{3,4}$ Patients with HF and/or COPD often wait to seek medical help in the early phases of deterioration, which usually leads to hospitalization as patients' conditions demand a more comprehensive medical procedure ${ }^{5,6}$. This is very costly and it is estimated that a $10 \%$ annual reduction of the number of COPD exacerbations occurring in Sweden would save approximately $€ 50$ million annually ${ }^{7}$.

New technology can be a barrier for elderly, severely ill patients with little or no experience of computers, tablets, smartphones, or technology in general; therefore, easeof-use concerning technology is essential ${ }^{8,9}$. To facilitate reporting, a digital pen technology has been used successfully in elderly palliative patients with end-stage cancer or advanced HF for the frequent assessment and reporting of symptoms, measurements, and intake of rescue medications to a HBHC unit ${ }^{10-13}$. 
During the last 10-15 years, there has been a growing number of original telehealth studies and reviews including patients with HF and/or COPD ${ }^{14-29}$. The focus of most of these studies has been to investigate if the use of a telehealth system can reduce disease related hospitalizations. The current study focused on all-cause hospitalizations, but also included emergency room visits, primary care visits, other outpatient care, and healthcare costs. Therefore, this study was designed to illustrate a more detailed analysis of costs and resources being used in a telehealth intervention. The aim in this study was to investigate the effects of the telehealth intervention on healthcare costs and the number of hospitalizations, as well as other care, in severely ill elderly patients with HF or COPD.

\section{Methods}

\section{Setting and usual care}

The eHealth Diary study was performed at the HBHC unit, Linköping University Hospital. The clinic provides specialized homecare to patients with advanced HF and/or COPD. The treatment period depends on the patient's condition, and, in usual care before the study, patients are discharged to primary care when they are stable with respect to HF/COPD. During the study period, as long as a patient participated in the eHealth Diary study, he/she was not discharged from the HBHC unit. However, all study patients had to consult primary care or other care providers for health conditions not related to HF or COPD.

\section{Study design}


The Health Diary system enables patients to report their daily health status, such as symptoms and measurement values, and intake of as-needed medication using a digital pen and a Health Diary paper form, and it supports professional caregivers in detecting early signs of patients' deterioration. The Health Diary system was designed, developed, tested, and implemented in collaboration with researchers, care providers at the HBHC unit and the software company Phoniro AB, Sweden. Assigned physicians and nurses at the HBHC unit supervised the system daily and provided most of the care needed. If a patient failed to send in his/her daily report the Health Diary system generated an SMS alarm the next morning. Likewise, if the care providers forgot to check and sign a patient report that had generated an alarm, the system sent an SMS alarm the next morning. Patient reports with values within the predetermined individual limits were to be checked and signed after a maximum of two weeks and if the care providers failed to do this the system generated alarms (both SMS and shown in the web application).

\section{Data collection}

Patients aged 65 years or older who visited the emergency department at Linköping University Hospital due to exacerbation for either COPD or HF with a history of at least two inpatient episodes the last twelve months, were invited to participate in the study. Patients with inpatient care at other departments at Linköping University Hospital who met the inclusion criteria could also be recruited, as could patients in primary care if their HF or COPD condition worsened, and if they met the inclusion criteria. Exclusion criteria comprised dementia, other cognitive impairment or psychotic illness, not able to understand the Swedish language, severe hearing loss, past surgery the last 6 months or planned within 6 months ahead, or other life-threatening illness. Patients were recruited 
until the number needed to study was reached ( $n=65$ in each group) due to the sample size calculation (a power of $80 \%$, alpha 0.05 and a difference of $15 \%$ between actual and expected outcomes). To ensure similar conditions for the study patients, an inclusion period of three years was used with a planned study period of twelve months for each patient, leading to a four-year study (November 2013-December 2017). Data on healthcare contacts (inpatient and outpatient) as well as direct healthcare costs were collected for each patient for one year or to date of death. All use of healthcare resources and costs, not only HF or COPD related, were considered, as HF and COPD are systemic disorders that can cause hospitalization for various reasons. The responsible physician identified patients' exacerbations and documented these in the electronic health record.

Using the unique 10-digit Swedish personal identity number, patient data were linked to the Care Data Warehouse in Östergötland (CDW) and Cost Per Patient database (CPP). The CDW includes all healthcare contacts (inpatient, outpatient, and primary care) for both private care and public care, and CPP includes costs for all contacts within public care.

For an adequate assessment of cost data, all costs collected from CPP were discounted with $3 \%$ per year up to the year 2017 and converted to euros $(1$ euro $=10$ SEK). Costs were missing in $14 \%$ of all contacts, mainly because of care given by private primary care providers. For these contacts, healthcare costs were imputed with the average cost calculated per contact type for existing data. Contact types with medical professionals were inpatient care, physician visit, district nurse visit, registered nurse visit, and visit to nurse assistant. Less than $1 \%$ of contacts with missing costs were related to inpatient care. 
Contacts with health services were grouped into inpatient care, emergency care, primary care, other outpatient care, and care provided by the HBHC unit. Patients receiving inpatient care and emergency care within two weeks before inclusion in the study were not included as they are major influences in the pre-study year and would, if they were included, overestimate the predicted expected value for the study year.

\section{Statistical analysis}

In this cohort study actual outcome data were compared to expected outcome data; the expected values were based on a 5-year history on the studied group. The primary outcomes were number of hospitalizations and total healthcare costs. The secondary outcomes were number of contacts with emergency care, primary care, other outpatient care, and the HBHC unit.

Expected use of healthcare resources and healthcare costs at study year were calculated using the generalized estimating equations (GEE) method. Poisson distribution with a log link was used for healthcare resources. For healthcare costs a gamma distribution or a normal distribution with a log link was chosen based on which one gave the best fit for actual data. For both healthcare resources and healthcare costs, repeated measures were considered and the covariance matrix was estimated using the robust estimator method. An unstructured or exchangeable working correlation matrix was chosen based on which one gave the best fit to actual data. To get the best fit to data, separate analysis models were used for HF and COPD groups. For each patient group, expected use of healthcare resources and healthcare costs was calculated for 1.

Inpatient care, 2. Emergency care, 3. Primary care, 4. Other outpatient care, and 5. Total healthcare resources/costs. For all models, the expected value at the study year was predicted using five-year data prior to the study year. To get a p-value for the ratio 
between the actual value and the expected value, a dichotomous variable with the value 1 at study year and 0 for non-study year was used in all models.

A t-test was used to test the proportion of death and follow-up time between the two patient groups. Chi-Square test was used for categorical data and median test to evaluate medians between groups.

As a sensitivity analysis, the number of hospitalizations and total healthcare costs were evaluated for patients completing the entire study period.

\section{Results}

Ninety-eight patients fulfilled the inclusion criteria and were asked to participate in the study. Three patients with HF and one patient with COPD declined to participate, giving a total of 94 patients: 58 patients with HF and 36 patients with COPD participated in the study which continued between November 2013 and December 2017 (Table 1). The HF group was $50 \%$ female and the median age was 84 years (range 65 to 100). The majority (97\%) of patients in the HF group were classified as NYHA 3B-4 of the New York Heart Association Functional Classification. The COPD group was $61 \%$ female and the median age was 74 years (range 65 to 86 ). The majority ( $72 \%$ ) of patients in the COPD group had very severe disease (stage IV) according to the GOLD-staging system. The proportion completing the study year was $54 \%(n=51) ; 31 \%(n=29)$ died and $15 \%(n=14)$ did not complete the study year for other reasons than death. There was no significant difference $(p=0.40)$ in the proportion of death between the HF group ( 28 $\%)$ and the COPD group (36 \%). There was no significant difference $(p=0.57)$ in the average follow-up time in years between the HF group (0.70) and the COPD group (0.65). 
For the HF group, the average use of healthcare resources without HBHC during the study year was 18.6 contacts (visits and hospitalizations) and this was significantly lower than expected $(p<0.001) 66 \%$ (Table 2, Fig. 1). All contact types, inpatient care, emergency care, primary care, and other outpatient care were significantly lower than expected. The average use of healthcare resources including HBHC was 55.8 contacts and this was $1 \%$ higher than expected, although this was not significant ( $p=0.94)$. The average total healthcare cost including $\mathrm{HBHC}$ was $€ 21515$; this was $20 \%$ lower than expected, although not significant ( $p=0.10)$ (Table 2, Fig.2).

For the COPD group, the average number of healthcare contacts without HBHC during the study year was 14.3 contacts and this was significantly lower than expected $(p<0.001) 60 \%$ (Table 2, Fig. 1). All contact types except other outpatient care were significantly lower than expected. The average number of contacts including HBHC was 71.2 contacts and this was twice as high as expected $(p=0.001)$. The average total healthcare cost including HBHC was $€ 33862$ and this was $25 \%$ higher than expected, although not significant $(p=0.20)$ (Table 2, Fig.2).

During the study year, the average cost for HBHC contributed with about $60 \%$ of total healthcare cost in both HF and COPD groups. The use of HBHC care was higher in the COPD group and contributed to $80 \%$ of total contacts compared with $67 \%$ in the HF group.

The sensitivity analysis showed that for patients completing the entire study period, the number of hospitalizations was 0.94 for HF and 1.16 for COPD. This was significantly lower than expected, with $67 \%$ in the HF group $(p<0.001)$ and $61 \%$ in the COPD group $(p=0.003)$. For patients completing the entire study period, total healthcare costs were $€ 25326$ in HF patients and $€ 42541$ in COPD patients. This was not 
significantly different from expected (expected value $=€ 23$ 841, $\mathrm{p}=0.72$ ) in HF patients, but in COPD patients the total healthcare cost was twice as high as expected (expected value $=€ 20981, \mathrm{p}<0.001)$; this was mainly due to additional HBHC utilization.

\section{Discussion}

The present study shows that daily telemonitoring for patients using an easy-to-handle digital pen and a Health Diary form, and a web-based application supervised by a specialized HBHC unit which is largely responsible for care provision, lowers the need of hospital care and primary care. Also, this study shows that total healthcare costs does not increase although patients frequently use care resources from the HBHC unit.

A Cochrane meta-analysis showed that HF-related hospitalization was significantly reduced using a telemonitoring system ${ }^{15,16}$. The present study demonstrates that the total number of hospitalizations were significantly lower than expected for both HF and COPD. The overall picture on how hospitalization is affected by a telemonitoring system in COPD patients seems to be ambiguous. This is illustrated by two systematic reviews showing that most studies found a positive result for the telemonitoring group, but also that some studies demonstrated a significantly increased number of hospitalizations in the intervention group. ${ }^{14,28}$ The use of different telehealth methodology is of importance in explaining the varying results, but also COPD patients are a heterogeneous group, and results may have been affected by different inclusion criteria in age, disease stage and need of palliative care ${ }^{14}$.

A Cochrane analysis for HF patients showed significantly reduced mortality for patients in the telemonitoring group ${ }^{15}$. In the present study, about one in three patients died within the study year, but the study design was not able to assess if this was lower than expected. A meta-analysis for COPD demonstrated no significant difference in 
mortality between a telephone support group and usual care ${ }^{28}$. The mortality in the present study was higher than in most published studies from the meta-analysis, but this is probably due to higher rates of inclusion for patients with advanced stages of disease. Unfortunately, we did not manage to recruit the number of patients estimated from the sample-size calculation within the inclusion period of three years. However, the effects were larger than we expected, resulting in significant outcomes.

Digital and other technology solutions in healthcare are important tools for the development of healthcare in general. There is a need to speed up the research in this area, otherwise there is a risk that decisions will be made without evidence from published peer review studies. One way to speed up research is to increase the use of existing historical data to estimate expected outcomes to compare with actual outcomes. The design of the eHealth Diary study used the study patients' historical data to estimate an expected outcome, which makes it possible to evaluate an intervention effect when randomization is not suitable. For this study there were two main reasons why this design was the most suitable. First, to ensure that the circumstances for the study patients were as similar as possible we needed to use one particular HBHC unit. Second, there was an ethical aspect of withholding treatment from suitable patients.

The mortality rate was high in this study. However, mortality was not an explanation of the decrease in the number of hospitalizations, as this outcome was similar when analyzing solely those who completed their 12-month study period. COPD patients who completed the entire study period accessed more HBHC care compared to the main analysis, which was not the case in HF patients. This might indicate that, despite the extensive telemonitoring, elderly patients with severe COPD are in need of extra personal care compared to HF patients. 
Results show that the intervention using an active telemonitoring system was effective for both HF and COPD patients in reducing hospitalizations for patients who died during the study period as well as for patients surviving the entire study period. Furthermore, the intervention reduced the number of visits to primary care, emergency care, and other outpatient care. The reduction in such health care contacts is presumably experienced as positive in elderly people's everyday life and that aspect will be explored in a further investigation of this study group.

The method using existing historical data instead of a randomized trial, in elderly patients with chronic disease, has many strengths. For instance, it is easier to initiate the study and a higher proportion of patients participate in the intervention because no control group is needed. However, an obvious limitation with this approach compared to a randomized trial is that mortality changes due to the intervention cannot be assessed, which makes the method inappropriate if mortality is the primary outcome.

One precondition for a telemonitoring system to contribute to a lowering in hospital care is that both professional care providers and patients are familiar with the technology and use it, i.e., they are compliant in sending (patients) and checking (care providers) reports. Nakamura et al. conclude that the speed of decision making and frequency of monitoring are the two most important factors for determining effectiveness of remote patient monitoring. With a high monitoring frequency the care providers will be able to capture changes in the patient's health status with greater sensitivity ${ }^{30}$. The patients in our study had little or no experience of using computers or smartphones but they quickly accepted and managed to handle the digital pen for daily reporting of their health status, and the HBHC unit managed to integrate the Health Diary system in daily clinical use. This was also the case in our previous pilot study 
with HF patients who continued to send in daily reports during several months without receiving any home visits from the care providers as long as their HF were in a stable phase ${ }^{10}$. In two earlier studies we let palliative cancer patients in their end-of-life use the same technology for frequent reporting (2-3 times per day) of symptoms and intake of pain medication ${ }^{12,31,32}$. One of the studies, an randomized controlled trial, comprised four palliative care centers in different areas in Sweden, and results showed a significant shorter median time span from reporting (patients) to noticing (care providers) in the intervention group (54 minutes) compared to the control group (47 hours). Thus, patients in the intervention group were more likely to receive a quicker medical response ${ }^{32}$. A patient receiving quicker medical response in his/her home has also an increased chance of remaining at home instead of going to the hospital.

The results from this study show that the Health diary intervention was successful in patients with advanced COPD or HF and other similar patient groups are likely to benefit from the intervention as well. In addition, the results may be generalized to other health care systems with a well-developed home care system.

\section{Conclusion}

A telemonitoring system, the Health Diary, combined with HBHC decreases the need for hospital care in elderly patients with advanced HF or COPD without increasing total healthcare costs. 


\section{Declaration of Conflicting Interests}

The Authors declares that there is no conflict of interest 


\section{References}

1. Zarrinkoub R, Wettermark B, Wandell P, et al. The epidemiology of heart failure, based on data for 2.1 million inhabitants in Sweden. Eur J Heart Fail. 2013; 15: 995-1002.

2. Backman H, Eriksson B, Ronmark E, et al. Decreased prevalence of moderate to severe COPD over 15 years in northern Sweden. Respiratory medicine. 2016; 114: 103-10.

3. Ponikowski P, Voors AA, Anker SD, et al. 2016 ESC Guidelines for the diagnosis and treatment of acute and chronic heart failure: The Task Force for the diagnosis and treatment of acute and chronic heart failure of the European Society of Cardiology (ESC). Developed with the special contribution of the Heart Failure Association (HFA) of the ESC. Eur J Heart Fail. 2016; 18: 891-975. 4. Global Initiative for Chronic Obstructive Lung Disease: Global strategy for the diagnoses, management and prevention of chronic obstructive pulmonary disease; 2018; https://goldcopd.org/wp-content/uploads/2018/02/WMS-GOLD-2018-Feb-Final-to-print-v2.pdf. 5. Korpershoek Y, Vervoort S, Nijssen L, Trappenburg J and Schuurmans MJ. Factors influencing exacerbation-related self-management in patients with COPD: a qualitative study. Int J Chron Obstruct Pulmon Dis. 2016; 11: 2977-90.

6. Patel H, Shafazand M, Schaufelberger M and Ekman I. Reasons for seeking acute care in chronic heart failure. Eur J Heart Fail. 2007; 9: 702-8.

$7 . \quad$ Nationella riktlinjer för vård vid astma och KOL; 2018. https://www.socialstyrelsen.se/Lists/Artikelkatalog/Attachments/20858/2018-1-36.pdf.

8. Foster MV and Sethares KA. Facilitators and barriers to the adoption of telehealth in older adults: an integrative review. Computers, informatics, nursing : CIN. 2014; 32: 523-33; quiz 34-5. 9. Hanson VL. Technology skill and age: what will be the same 20 years from now? Universal Access in the Information Society. 2011; 10: 443.

10. Lind L, Carlgren G and Karlsson D. Old-and With Severe Heart Failure: Telemonitoring by Using Digital Pen Technology in Specialized Homecare: System Description, Implementation, and Early Results. Computers, informatics, nursing : CIN. 2016; 34: 360-8.

11. Lind L, Berglund A, Berglund E, Bång M and Hägglund S. Effortless data capture for ambient e-services with digital pen and paper technology. In: Fransisco Milton Mendes Neto PFRN, (ed.). Designing Solution-Based Ubiquitous and Pervasive Computing : New Issues and Trends. Information Science Publishing/IGI Global, 2010, p. 24-43.

12. Lind L, Karlsson D and Fridlund B. Patients' use of digital pens for pain assessment in advanced palliative home healthcare. Int J Med Inform. 2008; 77: 129-36.

13. Lind L and Karlsson D. A system for symptom assessment in advanced palliative home healthcare using digital pens. Med Inform Internet Med. 2004; 29: 199-210.

14. Vitacca M, Montini A and Comini L. How will telemedicine change clinical practice in chronic obstructive pulmonary disease? Ther Adv Respir Dis. 2018; 12: 1753465818754778.

15. Dierckx R, Inglis SC, Clark RA, Prieto-Merino D and Cleland JG. Telemedicine in heart failure: new insights from the Cochrane meta-analyses. Eur J Heart Fail. 2017; 19: 304-6.

16. Inglis SC, Clark RA, Dierckx R, Prieto-Merino D and Cleland JG. Structured telephone support or non-invasive telemonitoring for patients with heart failure. Cochrane Database Syst Rev. 2015: CD007228.

17. Pinnock H, Hanley J, McCloughan L, et al. Effectiveness of telemonitoring integrated into existing clinical services on hospital admission for exacerbation of chronic obstructive pulmonary disease: researcher blind, multicentre, randomised controlled trial. BMJ. 2013; 347: f6070.

18. Jodar-Sanchez F, Ortega F, Parra C, et al. Implementation of a telehealth programme for patients with severe chronic obstructive pulmonary disease treated with long-term oxygen therapy. $J$ Telemed Telecare. 2013; 19: 11-7.

$19 . \quad H e n d e r s o n$ C, Knapp M, Fernandez JL, et al. Cost effectiveness of telehealth for patients with long term conditions (Whole Systems Demonstrator telehealth questionnaire study): nested economic evaluation in a pragmatic, cluster randomised controlled trial. BMJ. 2013; 346: f1035. 20. Steventon A, Bardsley M, Billings J, et al. Effect of telehealth on use of secondary care and mortality: findings from the Whole System Demonstrator cluster randomised trial. BMJ (Clinical research ed). 2012; 344: e3874-e.

21. Gellis ZD, Kenaley B, McGinty J, Bardelli E, Davitt J and Ten Have T. Outcomes of a telehealth intervention for homebound older adults with heart or chronic respiratory failure: a randomized controlled trial. Gerontologist. 2012; 52: 541-52. 

(COPD): an evidence-based analysis. Ont Health Technol Assess Ser. 2012; 12: 1-58.

23. Chau JP, Lee DT, Yu DS, et al. A feasibility study to investigate the acceptability and potential effectiveness of a telecare service for older people with chronic obstructive pulmonary disease. Int J Med Inform. 2012; 81: 674-82.

24. Antoniades NC, Rochford PD, Pretto JJ, et al. Pilot study of remote telemonitoring in COPD. Telemedicine journal and e-health : the official journal of the American Telemedicine Association. 2012; 18: 634-40.

25. McLean S, Nurmatov U, Liu JL, Pagliari C, Car J and Sheikh A. Telehealthcare for chronic obstructive pulmonary disease. Cochrane Database Syst Rev. 2011: CD007718.

26. Bolton CE, Waters CS, Peirce S, Elwyn G, Epsrc and Team MRCGC. Insufficient evidence of benefit: a systematic review of home telemonitoring for COPD. J Eval Clin Pract. 2011; 17: 1216-22.

27. Anker SD, Koehler F and Abraham WT. Telemedicine and remote management of patients with heart failure. Lancet. 2011; 378: 731-9.

28. Polisena J, Tran K, Cimon K, et al. Home telehealth for chronic obstructive pulmonary disease: a systematic review and meta-analysis. $J$ Telemed Telecare. 2010; 16: 120-7.

29. Polisena J, Tran K, Cimon K, et al. Home telemonitoring for congestive heart failure: a systematic review and meta-analysis. $J$ Telemed Telecare. 2010; 16: 68-76.

30. Nakamura N, Koga T and Iseki H. A meta-analysis of remote patient monitoring for chronic heart failure patients. $J$ Telemed Telecare. 2014; 20: 11-7.

31. Lind L, Karlsson D and Fridlund B. Digital pens and pain diaries in palliative home health care: professional caregivers' experiences. Med Inform Internet Med. 2007; 32: 287-96.

32. Lind L and Karlsson D. Electronic patient-reported symptom assessment in palliative end-of-life home care. Health Systems. 2013; 2: 171-80. 


\section{Tables}

Table 1. Baseline characteristics of 58 patients with HF and 36 patients with COPD

\begin{tabular}{|c|c|c|c|}
\hline & & & \\
\hline Age, median (range) & $84(65-100)$ & $74(65-86)$ & $<0.001$ \\
\hline Females, n (\%) & $29(50.0)$ & $22(61.1)$ & 0.29 \\
\hline Charlson's Comorbidity Index (CCI), mean (SD) & $4.9(2.3)$ & $3.4(2.0)$ & $<0.001$ \\
\hline Charlson's Comorbidity Index (CCI), n (\%) & & & $<0.001$ \\
\hline 0 & - & - & \\
\hline 1 & $4(6.9)$ & $5(13.9)$ & \\
\hline 2 & $4(6.9)$ & $9(25.0)$ & \\
\hline 3 & $6(10.3)$ & $12(33.3)$ & \\
\hline$\geq 4$ & $44(75.9)$ & $10(27.8)$ & \\
\hline GOLD stage, n (\%) & & & \\
\hline Stage I (Early) & - & - & - \\
\hline Stage II (Moderate) & - & $3(8.3)$ & - \\
\hline Stage III (Severe) & - & 7 (19.4) & - \\
\hline Stage IV (Very severe) & - & $26(72.2)$ & - \\
\hline GOLD grade, $n(\%)$ & & & \\
\hline Grade A-B & - & $1(2.8)$ & - \\
\hline Grade C-D & - & $0(0.0)$ & - \\
\hline Grade D & - & 35 (97.2) & - \\
\hline NYHA-class, n (\%) & & & \\
\hline NYHA 3 - 3a & $2(3.4)$ & - & - \\
\hline NYHA 3b & $44(75.9)$ & - & - \\
\hline NYHA 4 & $12(20.7)$ & - & - \\
\hline
\end{tabular}

HF: Heart Failure; COPD: Chronic obstructive pulmonary disease 
Table 2 Observed and expected mean values for use of healthcare resources and costs (€) in 58 patients with HF and 36 patients with COPD

\begin{tabular}{|c|c|c|c|c|c|c|c|c|}
\hline \multirow[b]{2}{*}{ Healthcare resources } & \multicolumn{4}{|c|}{$\mathrm{HF}$} & \multicolumn{4}{|c|}{ COPD } \\
\hline & Observed & Expected & Observed/Expected & $P$-value & Observed & Expected & Observed/Expected & $P$-value \\
\hline Inpatient care & 0.8 & 3.1 & $26 \%$ & $<0.001$ & 1.2 & 3.1 & $39 \%$ & $<0.001$ \\
\hline Emergency care & 0.8 & 4.8 & $17 \%$ & $<0.001$ & 1.2 & 3.9 & $31 \%$ & $<0.001$ \\
\hline Primary care & 13,0 & 35.9 & $36 \%$ & $<0.001$ & 6.4 & 16.4 & $39 \%$ & 0.001 \\
\hline Other outpatient care & 4.1 & 7.8 & $53 \%$ & $<0.001$ & 5.6 & 8.6 & $65 \%$ & 0.11 \\
\hline Total contacts - HBHC & 18.6 & 55.3 & $34 \%$ & $<0.001$ & 14.3 & 35.8 & $40 \%$ & $<0.001$ \\
\hline Total contacts $+\mathrm{HBHC}$ & 55.8 & 55.3 & $101 \%$ & 0.94 & 71.2 & 35.8 & $199 \%$ & $<0.001$ \\
\hline \multicolumn{9}{|l|}{ Healthcare costs } \\
\hline Inpatient care & 4823 & 17604 & $27 \%$ & $<0.001$ & 10516 & 23945 & $44 \%$ & 0.01 \\
\hline Emergency care & 410 & 2316 & $18 \%$ & $<0.001$ & 615 & 2133 & $29 \%$ & $<0.001$ \\
\hline Primary care & 1707 & 5329 & $32 \%$ & $<0.001$ & 683 & 2946 & $23 \%$ & $<0.001$ \\
\hline Total costs + HBHC & 21515 & 26798 & $80 \%$ & 0.10 & 33862 & 27016 & $125 \%$ & 0.20 \\
\hline
\end{tabular}

HF: Heart Failure; COPD: Chronic obstructive pulmonary disease; HBHC: Specialized hospital-based home care; bold=significant result

The sum of contacts/costs for each type does not equal the total contacts/costs because of separate statistical models 


\section{Figures}

Figure 1

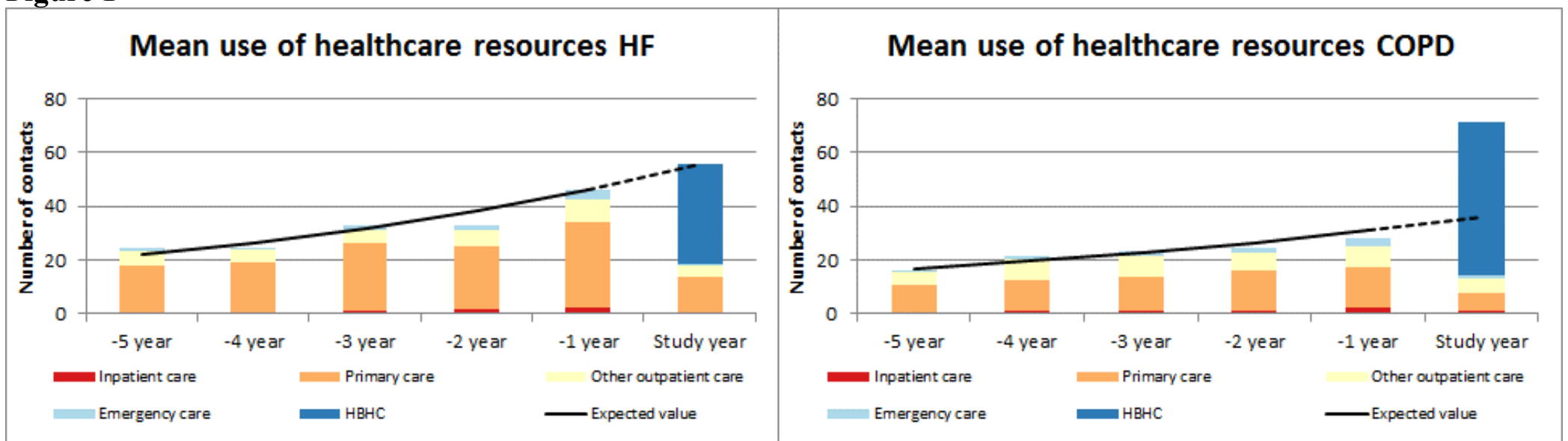

HF: Heart failure; COPD: Chronic obstructive pulmonary disease; HBHC: Specialized hospital-based home care; dotted line is expected value for the study year, based on previous use of resources (five years)

Figure 2

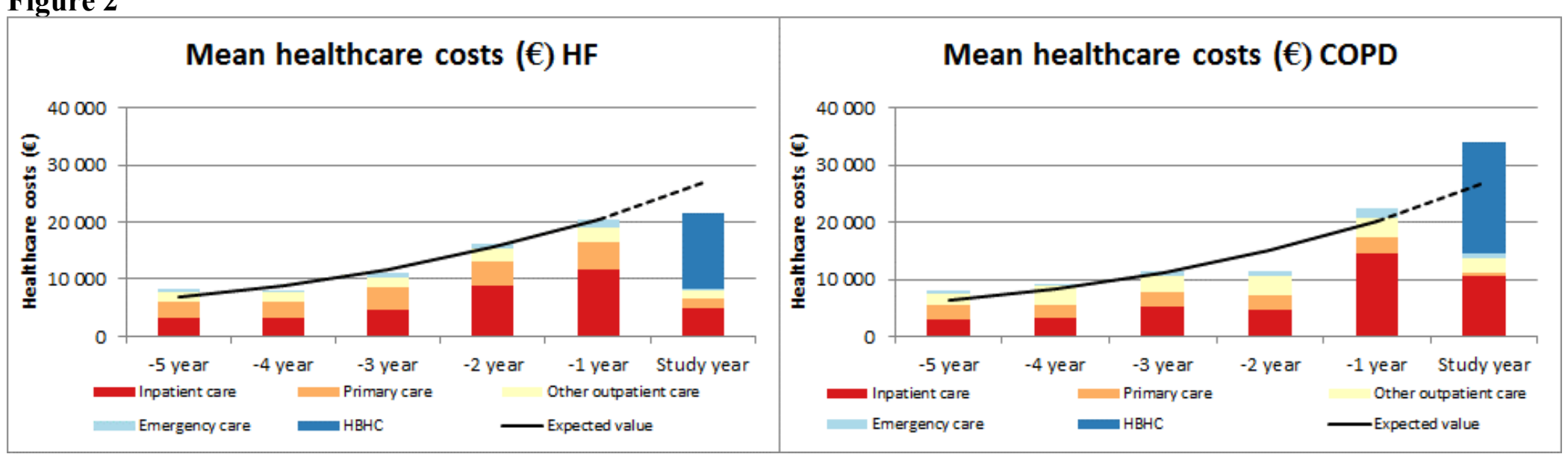

HF: Heart failure; COPD: Chronic obstructive pulmonary disease; HBHC: Specialized hospital-based home care; dotted line is expected value for the study year, based on previous costs (five years) 